\title{
Cellular footprints of plant evolution-news from the blepharoblast
}

\author{
Peter Nick
}

Published online: 6 August 2008

(C) Springer-Verlag 2008

The microtubular cytoskeleton of higher plants diverges considerably from its animal counterpart. This divergence involves a fundamentally different organization with microtubule arrays, which are specific to higher plants, such as cortical microtubules or the phragmoplast. On the other hand, centrioles, which are central organizers of microtubules in cells of animals and lower plants, have been progressively reduced in the course of plant evolution, and are now absent, as in most seed plants. The role of centrioles as major microtubule-organizing centers (MTOC) apparently has been taken over by the nuclear envelope. However, the spermatogenous cells of pteridophytes and some of the primitive gymnosperms are still endowed with centriolar structures, which is probably related to the fact that the spermatozoids move by flagella. In other words: these specialized cells represent something like a "missing link" between the ancestral situation, where microtubules are nucleated from persistent microtubule rods that are organized in a specialized organelle, and a derived situation, where microtubules emerge from more or less diffuse MTOCs.

In the pteridophytes, the centriolar structure is known as the blepharoplast and arises in the last two division cycles. It organizes the interphase array of microtubules along with the spindle for the two final divisions. Prior to each of these mitotic events, the blepharoplast divides itself and moves to the poles, serving as the focus for an astral spindle. After the final division, it re-organizes to a much looser structure

\section{P. Nick $(\bowtie)$}

Botanical Institute 1,

Kaiserstr. 2,

76128 Karlsruhe, Germany

e-mail: peter.nick@bio.uni-karlsruhe.de and basal bodies are produced that will eventually organize the sperm flagellar apparatus.

The work by Vaughn and Bowling in the present issue highlights this evolutionarily interesting transition. Using spermatogenous cells of the fern Ceratopteris richardii, they investigate the effect of transient elimination of microtubules. They use oryzalin, an inhibitor that sequesters tubulin heterodimers from being incorporated into the growing microtubule, such that microtubules shorten and eventually disappear as consequence of their innate turnover. The blepharoplast contains barrel-like structures that serve as centriolar templates embedded in an electronopaque pericentriolar matrix. Upon treatment with oryzalin they observe that this pericentriolar material forms clear gaps that correspond in their dimension to the typical microtubular diameter and stain with $\gamma$-tubulin antibodies. Despite the absence of microtubules, the division of the blepharoblast is observed to continue - this means that the division of the MTOC seems to be independent of the presence of microtubules. This represents an evolutionary "modern" trait heralding the situation in higher plant cells, where microtubule arrays cycle although there are no centriolar microtubule asters. The gaps might be morphological manifestations of the $\gamma$-tubulin ring complexes that nucleate microtubules in different phyla. After washout, the microtubule arrays showed malformations or multiplications that mirrored the behaviour of the blepharoblasts during drug treatment. Therefore, in terms of cell biology, the spermatogenous cells combines an evolutionarily ancient situation, namely, dependence of microtubule organization from a centriolar organelle, with an evolutionarily derived trait, independence of microtubule organizers from preexisting microtubules. These cells are therefore exciting models to follow the cellular footprints of plant evolution. 